Christos Koulamas

Department of Decision Sciences and Information Systems

Florida International University

Miami, FL 33199

Tel. (305) 348-3309

Fax: (305) 348-4126

(March, 2004) 


\title{
INVERSE SCHEDULING WITH CONTROLLABLE JOB PARAMETERS
}

\begin{abstract}
Scheduling problems deal with how to sequence a list of jobs with the objective of minimizing some measurement of the job completion times. Inverse scheduling problems assume that a job sequence is given and the objective is to determine the minimal perturbation to the job parameters (e.g. processing times) so that the given sequence becomes optimal with respect to a pre-selected objective function. The objective of this paper is to study inverse scheduling problems. It will be shown that these problems can be formulated as linear programming (LP) problems even when, in some cases, the corresponding forward scheduling problems are not solvable in polynomial time. Several applications are discussed, including the generation of benchmark optimal solutions for NP-hard forward scheduling problems.
\end{abstract}

Keywords: scheduling/single-machine, flow shop, linear programming.

\section{INTRODUCTION}

Inverse optimization is concerned with determining the minimum perturbation to the cost parameter vector of a problem, so that a given feasible solution becomes optimal. There are numerous applications of inverse optimization in the areas of geophysical sciences, medical imaging and traffic equilibrium problems among others. Ahuja and Orlin (2001) study a wide variety of inverse optimization problems and their applications, including general linear programming, shortest path, minimum cost and assignment 
problems among others. The objective of this paper is to extend the inverse optimization approach to scheduling problems and discuss applications of inverse optimization specific to scheduling problems. The traditional forward scheduling problem deals with how to sequence a given list of jobs with the objective of minimizing some measurement of the job completion times. If the chosen objective is a non-decreasing function of the job completion times, it is called a regular measurement. The corresponding inverse scheduling problem assumes that a job sequence is given and the objective is to determine the minimal perturbation to the job parameters (e.g. processing times) so that the given sequence becomes optimal with respect to the pre-selected objective.

The main application of inverse scheduling problems is in situations where the job parameters can be negotiated between the production shop and its customers. The original job parameters are the ones preferred by the customers; the corresponding perturbed values are the ones proposed by the shop management, so that the shop can follow a preset master production schedule and at the same time the applicable objective function is minimized. Other application of inverse scheduling problems can be found in nearly on-line systems where jobs must be sequenced in the order they become available but, unlike purely on-line systems, there is some control over the job parameters. More specifically, in nearly on-line systems, the scheduler obtains detailed information about a job's parameters when it is released to the system. The scheduler can then negotiate the actual values of these parameters with the customers in order to maintain the optimality of the current schedule even after the newly arrived job is appended to it. The application of inverse scheduling will determine the minimum required perturbation in the aforementioned job parameters so that the optimality of the schedule is maintained. 
The objective of this paper is to study inverse scheduling problems. It will be shown that these problems can be formulated as linear programming (LP) problems even when, in some cases, the corresponding forward scheduling problems are not solvable in polynomial time. Specific applications of inverse scheduling (other than the ones mentioned above) will also be presented like the use of inverse scheduling to generate benchmark optimal solutions for certain NP-hard forward scheduling problems.

A related subject in the scheduling literature is the sequencing problem with controllable job parameters. Vickson (1980) considered a single-machine problem with controllable job processing times and his objective was to determine a job sequence and actual processing times that minimize the schedule cost. The survey by Nowicky and Zdrzalka (1990) reviews subsequent work with controllable job processing times. Panwalkar et al. (1982), Chand and Chhajed (1992), and the references therein consider scheduling problems with controllable job due dates. More recent contributions on scheduling with controllable job parameters include the work of Trick (1994) on scheduling multiple variable-speed machines and the work of Alidaee and Kochenberger (1996). The objective in these papers is to determine job parameter values and job sequences to minimize some function of the job due dates and/or completion times. The main difference between the above forward scheduling problems and the inverse scheduling problem studied in this paper is that the latter assumes a given job sequence and is concerned with converting that sequence to an optimal one by minimally perturbing the controllable job parameters.

There is also literature on other inverse optimization problems, like inverse linear programming (Ahuja and Orlin, 2001), inverse assignment and minimum cost flow 
problems (Zhang and Liu, 1996), inverse minimum cut problems (Yang et al., 1997), and inverse shortest path problems (Xu and Zhang, 1995) among others.

To our knowledge, there is no literature on inverse scheduling problems except for the literature mentioned above on the related forward scheduling problems with controllable job processing times and/or due dates.

The rest of the paper is organized as follows. Section 2 is concerned with singlemachine inverse scheduling problems. Multiple-operation (flow shop) inverse scheduling problems are considered in section 3. Some concluding remarks are stated in section 4.

\section{SINGLE-MACHINE INVERSE SCHEDULING PROBLEMS}

Consider an arbitrary $n$-job sequence on a single machine. Each job $j$, $j=1, \ldots, n$ has a processing time $p_{j}$ associated with it. Let $C_{j}=\sum_{k=1}^{j} p_{k}$ be the completion time of job $j$ in this arbitrary sequence. We consider the problem of converting this arbitrary sequence to an optimal one with respect to the total $\sum_{j=1}^{n} C_{j}$ (or average, $\bar{C}$ ) job completion time criterion. Using the well-known three-field notation for defining scheduling problems, the corresponding forward problem can be defined as $1 / / \bar{C}$, where the first field defines the machine environment, the middle field is reserved for special job parameters/problem characteristics and the third field denotes the objective function. In the rest of the paper, we will enter $I N V$ in the middle field to define inverse scheduling problems. It is well known (Smith, 1956) that the $1 / / \bar{C}$ problem is solved optimally by sequencing the jobs in the shortest processing time (SPT) 
order. Consequently, the $1 / I N V / \bar{C}$ problem is solved by determining the minimum total perturbation to the job processing times so that a given sequence is converted into an SPT sequence. We formulate the $1 / I N V / \bar{C}$ problem as a mathematical programming problem ( $L P 1 a)$ :

Problem LPla:

$$
\min \sum_{j=1}^{n} c_{j}\left|p_{j}^{*}-p_{j}\right|
$$

subject to:

$$
0 \leq p_{1}^{*} \leq p_{2}^{*} \leq \ldots \leq p_{n}^{*}
$$

where $p_{j}^{*}$ is the new minimally perturbed processing time of job $j$ and $c_{j}$ is the unit perturbation cost of $p_{j}, j=1, \ldots, n$. In the rest of the paper, we use the superscript "**" to define perturbed job parameters. The above formulation is flexible enough, since it allows for an arbitrary job processing time $p_{j}$ not be perturbed at any cost by setting the corresponding unit cost $c_{j}$ to a very large number. $L P 1 a$ is not a linear programming problem but can be converted into one by using a standard transformation. It is well known that minimizing $\left|p_{i}^{*}-p_{i}\right|$ is equivalent to minimizing $x_{i}+y_{i}$, subject to $p_{i}^{*}-p_{i}=x_{i}-y_{i}, \quad x_{i} \geq 0, y_{i} \geq 0$. Using this transformation, problem LPla can be restated as follows:

Problem LP1b:

$$
\min \sum_{i=1}^{n} c_{i} x_{i}+\sum_{i=1}^{n} c_{i} y_{i}
$$

subject to: 


$$
\begin{aligned}
& p_{i}^{*}-p_{i}=x_{i}-y_{i}, i=1, \ldots, n \\
& 0 \leq p_{1}^{*} \leq p_{2}^{*} \leq \ldots \leq p_{n}^{*} \\
& x_{i} \geq 0, y_{i} \geq 0, i=1, \ldots, n
\end{aligned}
$$

As an application, we solved a four-job $1 / I N V / \bar{C}$ problem with $\left\langle p_{1}, p_{2}, p_{3}, p_{4}\right\rangle=\langle 6,4,8,2\rangle$ and $\left\langle c_{1}, c_{2}, c_{3}, c_{4}\right\rangle=\langle 1,1,1,1\rangle$ using formulation $L P 1 b$. The resulting $L P 1 b$ solution was $\left\langle p_{1}^{*}, p_{2}^{*}, p_{3}^{*}, p_{4}^{*}\right\rangle=\langle 6,6,8,8\rangle$.

Formulation $L P 1 b$ can also be used to solve other inverse single-machine sequencing problems that require the perturbation of just one job parameter. Furthermore, the $L P 1 b$ formulation can be used to solve a broad category of inverse single-machine scheduling problems as long as the optimal sequence for the corresponding forward problems can be obtained by performing a sorting operation on a single job parameter. Such problems include (among others) the problem of minimizing the makespan $C_{\max }=\max _{j=1, \ldots, n}\left\{C_{j}\right\}$ in the presence of unequal job release times $r_{j}$, (the $1 / r / C_{\max }$ problem is solved optimally by sequencing the jobs in non-decreasing $r_{j}$ order) and the symmetric $1 / q / C_{\max }$ problem of minimizing the makespan $C_{\max }=\max _{j=1, \ldots, n}\left\{C_{j}+q_{j}\right\}$ in the presence of jobs delivery times (tails) $q_{j}$ when all jobs are available at time zero (the $1 / q / C_{\max }$ problem is solved optimally by sequencing the jobs in non-increasing $q_{j}$ order). The corresponding inverse scheduling problems can be formulated along the lines of the $L P 1 b$ formulation by minimizing the total perturbation of the job release times $r_{j}$ (in the $1 / r, I N V / C_{\max }$ case) and the total perturbation of the job delivery times $q_{j}$ (in the $1 / q, I N V / C_{\max }$ case) 


\section{FLOW SHOP INVERSE SCHEDULING PROBLEMS.}

In this section, we turn our attention to multiple operation scheduling problems. Their main characteristic is that each job requires $m \geq 2$ operations to be processed in any order (open shops) or in the same pre-specified order for all jobs (flow shops), or in some predetermined order that differs among jobs (job shops). The most commonly used optimization criterion in multiple operation scheduling problems is the minimization of the makespan $C_{\max }=\max _{j=1, \ldots, n}\left\{C_{m j}\right\}$ where $C_{m j}$ is the completion time of the $m$ (last) operation of job $j, j=1, \ldots, n$.

In the rest of this section, we will study two representative inverse scheduling shop problems, namely, the two-machine flow shop $F 2 / I N V / C_{\max }$ and the $m$-machine flow shop $F m / I N V / C_{\max }$ problems. These two problems are representative of the two general categories of inverse scheduling problems based on the complexity of the corresponding forward problems. In the $F 2 / I N V / C_{\max }$ case, the corresponding forward problem $F 2 / / C_{\max }$ is polymomially solvable by the $O(n \log n)$ Johnson's (1954) algorithm, while in the $\mathrm{Fm} / \mathrm{INV} / \mathrm{C}_{\max }$ case, the corresponding forward problem $F m / / C_{\max }$ is NP-hard (Garey et al., 1976).

\subsection{The $F 2 / I N V / C_{\max }$ problem}

Let $a_{j}, b_{j}$ be the processing time of job $j, j=1, \ldots, n$ for the first and second operations (stages) respectively. Johnson's algorithm produces an optimal permutation schedule (with the same job sequence in both stages) for $F 2 / / C_{\max }$ by sequencing first 
all jobs with $a_{j} \leq b_{j}$ in non-decreasing $a_{j}$ order followed by the remaining jobs in nonincreasing $b_{j}$ order. We first derive an LP formulation $(L P 2 a)$ for the $F 2 / I N V / C_{\max }$

problem assuming that job $k$, the last job in the given sequence with $a_{k}^{*} \leq b_{k}^{*}$ after the perturbation on the job processing times is applied, (to be called the critical job from now on), is known; let $c_{1 i}, c_{2 i}$ be the unit perturbation cost of $a_{i}, b_{i}$ respectively, $i=1, \ldots, n$; LP5 $a$ is as follows:

Problem LP2a:

$$
\min \sum_{i=1}^{n} c_{1 i}\left|a_{i}^{*}-a_{i}\right|+\sum_{i=1}^{n} c_{2 i}\left|b_{i}^{*}-b_{i}\right|
$$

subject to:

$$
\begin{aligned}
& 0 \leq a_{1}^{*} \leq a_{2}^{*} \leq \ldots a_{k}^{*} \\
& b_{k+1}^{*} \geq b_{k+2}^{*} \geq \ldots \geq b_{n}^{*} \geq 0 \\
& a_{j}^{*} \leq b_{j}^{*}, 1 \leq j \leq k \\
& a_{j}^{*} \geq b_{j}^{*}, k<j \leq n
\end{aligned}
$$

Problem $L P 2$ must be solved $n$ times since there are $n$ possible values for the critical job $k$.

\subsection{The $F m / I N V / C_{\max }$ problem}

Let $p_{i j}$ be the processing time of job $j$ on machine (stage) $i$ in the $F m / / C_{\max }$ problem. In order to derive an LP formulation for the $F m / I N V / C_{\max }$ problem, we will utilize a polynomially solvable case of the $F m / / C_{\max }$ problem. Specifically, we will consider the case of a minimal (maximal) machine, where all jobs have their minimum (maximum) processing time on the same machine, under some additional conditions on 
the remaining machines (Chin and Tsai, 1981). The most general machine configuration proposed by Chin and Tsai (1981) that can be solved in polynomial time is the $E_{1} E_{1} \ldots E_{1} E_{2} E_{2} \ldots E_{2}$ configuration; in that configuration, $p_{1 j}=p_{2 j}=\ldots=p_{l j}$ and $p_{l+1, j}=p_{l+2, j}=\ldots=p_{m j}$ for all jobs $j=1, \ldots, n$, and for some machine $l=1, \ldots, m-1$; machine $l$ will be called the switchover machine from now on. An optimal makespan sequence for the above problem is obtained by applying Johnson's rule to machines $l, l+1$. We shall exploit this special case to derive an LP formulation for the $\mathrm{Fm} / \mathrm{INV} / \mathrm{C}_{\max }$ problem by generalizing formulation $L P 2$ to the present situation. This generalization requires the definition of $n^{2} m(m-1)$ variables to account for all $n(m-1)$ possible combinations of the critical job $k$ and the switchover machine $l$ respectively; let $p_{i j}^{l k^{*}}$ be the perturbed processing times of job $j$ on machine $i$ when job $k$ is the critical job and machine $l$ is the switchover machine. The $k, l$ combination that results in the minimum total job processing time perturbation can be retrieved by a postoptimization minimum operation among the $n(m-1)$ summands in the two outer summations in the optimal objective function value of the following formulation:

Problem LP3:

$$
\min \sum_{l=1}^{m-1} \sum_{k=1}^{n}\left\{\sum_{j=1}^{n} \sum_{i=1}^{m} c_{i j}\left|p_{i j}^{l k^{*}}-p_{i j}\right|\right\}
$$

subject to:

$$
\begin{aligned}
& 0 \leq p_{l 1}^{l k^{*}} \leq p_{l 2}^{l k^{*}} \leq \ldots p_{l k}^{l k^{*}}, k=1, \ldots, n, l=1, \ldots, m-1 \\
& p_{l+1, k+1}^{l k^{*}} \geq p_{l+1, k+2}^{l k^{*}} \geq \ldots \geq p_{l+1, n}^{l k^{*}} \geq 0, k=1, \ldots, n, l=1, \ldots, m-1 \\
& p_{l j}^{l k^{*}} \leq p_{l+1, j}^{l k^{*}}, 1 \leq j \leq k, k=1, \ldots, n, l=1, \ldots, m-1
\end{aligned}
$$




$$
\begin{aligned}
& p_{l j}^{l k^{*}}>p_{l+1, j}^{l k^{*}}, k<j \leq n, k=1, \ldots, n, l=1, \ldots, m-1 \\
& p_{i j}^{l k^{*}}=p_{l j}^{l k^{*}}, j=1, \ldots, n, i=1, \ldots, l-1, k=1, \ldots, n, l=1, \ldots, m-1 \\
& p_{i j}^{l k^{*}}=p_{l+1, j}^{l k^{*}}, j=1, \ldots, n, i=l+2, \ldots, m, k=1, \ldots, n, l=1, \ldots, m-1
\end{aligned}
$$

where $c_{i j}$ is the unit perturbation cost of $p_{i j}$.

\section{CONCLUDING REMARKS}

We studied several machine inverse scheduling problems. We showed that these problems can be formulated as linear programming problems even when the corresponding forward problems are not solvable in polynomial time. In the latter case we identified additional conditions on the job parameters values that make the corresponding forward problems solvable in polynomial time and subsequently incorporated these conditions in our linear programming formulations. In those cases, our formulations can be used to generate benchmark optimal solutions for the corresponding forward problems which in turn can be used to evaluate the performance of enumerative algorithms for the aforementioned forward problems. 


\section{REFERENCES}

Ahuja, R.K., and J.B. Orlin, 2001, "Inverse Optimization", Operations Research, 49, 771-783.

Alidaee, B. and G. A. Kochenberger, 1996, "A Framework for Machine Scheduling Problems with Controllable Processing Times", Production and Operations Management, 5, 391-405.

Chand, S., and D. Chhajed, 1992, "A Single Machine Model for Determination of Optimal Due Dates and Sequence", Operations Research, 40, 596-602.

Chin, F.Y., and L. Tsai, 1981, “On J-maximal and J-minimal flow shop schedules”, Journal of ACM, 28, 462-476.

Garey, M.R., D.S. Johnson, and R. Sethi, 1976, “The complexity of flowshop and jobshop scheduling", Mathematics of Operations Research, 1, 117-129.

Johnson, S.M., 1954, "Optimal two- and three-stage production schedules with setup times included", Naval Research Logistics Quarterly, 1, 61-68.

Nowicki, E., and S. Zdrzalka, 1990, "A survey of results for sequencing problems with controllable processing times", Discrete Applied Mathematics, 26, 271-287.

Panwalkar, S.S., M.L. Smith, and A. Seidmann, 1982, "Common Due Date Assignment to Minimize Total Penalty for the One Machine Scheduling Problem", Operations Research, 30, 391-399.

Smith, W.E., 1956, "Various optimizers for single-stage production", Naval Research Logistics Quarterly, 3, 59-66.

Trick, M., 1994, "Scheduling Multiple Variable-Speed Machines", Operations Research, 42, 234-248. 
Vickson, R.G., 1980, “Choosing the job sequence and processing times to minimize total processing plus flow cost on single machine”, Operations Research, 28, 11551167.

$\mathrm{Xu}, \mathrm{S}$., and J. Zhang, 1995, “An inverse problem of the weighted shortest path problem”, Japanese Journal of Industrial Applied Mathematics, 12, 47-59.

Yang, C.J., J. Zhang, and Z. Ma, 1997, "Inverse maximum flow and minimum cut problems", Optimization, 40, 147-170.

Zhang, J., and Z. Liu, 1996, “Calculating some inverse lineal programming problem”, Journal of Computational Applied Mathematics, 72, 261-273. 